\title{
Estimating Mechanical Brain Tissue Properties with Simulation and Registration
}

\author{
Grzegorz Soza $^{1}$, Roberto Grosso ${ }^{1}$, Christopher Nimsky ${ }^{2}$, Guenther Greiner ${ }^{1}$, \\ and Peter Hastreiter ${ }^{1,2}$ \\ 1 Computer Graphics Group, University of Erlangen-Nuremberg, \\ Am Weichselgarten 9, 91058 Erlangen, Germany \\ soza@cs.fau.de \\ ${ }^{2}$ Neurocenter, Department of Neurosurgery, University of Erlangen-Nuremberg
}

\begin{abstract}
In this work a new method for the determination of the mechanical properties of brain tissue is introduced. Young's modulus $E$ and Poisson's ratio $\nu$ are iteratively estimated based on a finite element model for brain shift and on the information contained in pre- and intraoperative MR data after registration. In each iteration, a 3D dataset is generated according to the displacement vector field resulting from a numerical simulation of the intraoperative brain deformation. This reconstruction is parametrized by elastic moduli of tissue. They are automatically varied in order to achieve the best correspondence between the grey value distribution in the reconstructed image and the intensity entropy in the MR image of the brain undergoing deformation. This work contributes to the difficult problem of defining correct mechanical parameters to perform reliable model calculations of brain deformation. Proper boundary conditions that are crucial in this context are also addressed.
\end{abstract}

\section{Introduction}

Computer assisted systems for medical diagnosis, surgery training and therapy require precise computational methods for modeling the deformation of soft tissue. Within the context of neurosurgery, brain shift has been described with various physical and mathematical models. However, even sophisticated algorithms are limited without precise information about the elastic tissue parameters.

Miller et al. validated the mechanical properties of swine brain tissue obtained in vitro in a series of experiments in vivo 9. Simulated forces were $31 \%$ lower than those recorded in experiments, which can be, among others, attributed to the fact that the mechanical properties of deceased tissue are different from those of the tissue in vivo.

Biomechanical characterization of living soft tissues has been a subject of intensive investigations in recent years. Different diagnostic imaging modalities have been applied in numerous experiments in order to measure the response of the tissue to various types of loadings. In this context, mainly magnetic resonance imaging (MRI) and ultrasound (US) have been investigated.

Tissue elasticity imaging methods based on US can be divided into two main groups. US waves are considered for detecting internal tissue motion resulting 
from a static mechanical stimulus [4] (here in vitro). Other researchers applied US to observe the behavior of tissue under a low-frequency vibration [12. MRI has been applied, among others, in investigations of the shear modulus of the brain in vivo [5]. A very comprehensive survey of similar works discussing methods for measuring tissue stiffness is given in [10]. Low availability and difficulties with the integration in clinical practice are, however, the main disadvantages of such elasticity imaging techniques.

At the same time, there have been investigations focusing on the implicit estimation of the mechanical properties of the tissue. Various relations between Lamé constants which were reported in the literature for human brain tissue were presented in [1. The influence of different tissue elasticity values on the accuracy of the simulation was investigated in a comparative study of biomechanical breast models 16. Also, within the context of mammography, regional measures of image similarity were used by Miga et al. in a function minimization framework to reconstruct elasticity images of tissue stiffness [6]. In that work Young's moduli were analyzed for 2D cross sectional MR slice of breast tissue.

However, it is crucial for the simulation to incorporate both, Young's modulus $E$ and Poisson's ratio $\nu$ in the reconstructed mechanical properties of tissue since their combination is very important for the behavior of the simulation model. The presented method accounts for this issue and calculates both elastic moduli based on registering the result of a biomechanical simulation and corresponding intraoperative MR data. Furthermore, the approach has been applied for the first time to a $3 \mathrm{D}$ brain deformation model.

\section{Method}

\subsection{Model for Parameter Estimation}

The model for the automatic determination of the Young's modulus $E$ and the Poisson's ratio $\nu$ for the brain tissue is based on an iterative process where a biomechanical model of brain shift interacts with the information extracted from MRI head data acquired before and during craniotomy. In each iteration, a volumetric dataset is reconstructed according to the displacement vector field obtained as a result of the numerical simulation of the intraoperative brain deformation (see Section 2.3). This reconstruction is parametrized by the elastic moduli of tissue $E$ and $\nu$. The main idea of the presented approach for the estimation of the mechanical properties of the brain tissue is to vary these parameters in such a way that the correspondence between the reconstructed volume and the intraoperative dataset is maximized.

The basis for this is the normalized mutual information (NMI) [15] which relates the grey value distributions in both datasets in terms of entropy. We expressed this measure as a function of the elastic moduli $E$ and $\nu$ which are free parameters in the optimization procedure. The similarity function denoted by $\mathbf{S}_{\text {sim,intra }}$ is formulated as 


$$
\begin{aligned}
\mathbf{S}_{\text {sim,intra }}(E, \nu)= & N M I_{\text {sim,intra }}(E, \nu)+\alpha_{0}<E_{\text {low }}, E_{\text {high }}>(E)+ \\
& \alpha_{1}<\nu_{\text {low }}, \nu_{\text {high }}>(\nu),
\end{aligned}
$$

with $\alpha_{0}$ representing a penalty term which is defined by

$$
\alpha_{0}<E_{\text {low }}, E_{\text {high }}>(E)=\left\{\begin{array}{rl}
c_{0} & : E \notin<E_{\text {low }}, E_{\text {high }}>, c_{0} \leq 0 \\
0 & : \quad E \in<E_{\text {low }}, E_{\text {high }}>
\end{array} .\right.
$$

The search space for $E$ can be constrained by setting the upper and low limits $E_{\text {high }}$ and $E_{\text {low }}$ and specifying the penalty constant $c_{0}$. Thus, expertise about the reasonable interval for this elasticity parameter is incorporated into the optimization process. This reduces the number of iterations required for the determination of the optimal values for $E$. The same considerations hold for $\alpha_{1}$ and $\nu$. Note, that this restriction term can be skipped by setting $c_{0}=0$, thus allowing the optimization in the whole parameter space.

\subsection{Numerical Model}

During optimization, the similarity between the intraoperative MRI and the volume resulting from the physically-based simulation is computed in each iteration. Generally, the underlying mathematical model of the soft tissue deformation can be chosen arbitrarily. For this purpose, in this work we considered a simplified set of equations describing the behavior of poroelastic materials under load [13]. Thereby, the brain is assumed to be a linearly elastic medium saturated by a viscous fluid. The constitutive equations

$$
\begin{aligned}
-\frac{\mu}{1-2 \nu} \nabla(\nabla \cdot \mathbf{u}(x, t))-\mu \Delta \mathbf{u}(x, t)+\alpha \nabla p(x, t) & =\mathbf{f}(x, t) \\
\frac{\partial}{\partial t}\left(d_{0} p(x, t)+\alpha \nabla \cdot \mathbf{u}(x, t)\right)-\nabla \cdot k \nabla p(x, t) & =h(x, t)
\end{aligned}
$$

are coupled. This means that the compression of the solid enhances the fluid flow due to an increased pore pressure. On the other hand, an increasing pore pressure results in stress in the deformable solid matrix. In the equations (3a) and ( $(3 \mathrm{~b})$, fluid pressure is referred to by $p(x, t)$ and the displacement vector by $\mathbf{u}(x, t)$. The parameter $t$ expresses the time dependence of the system. Parameters corresponding to the elastic properties of the tissue are Poisson's ratio $\nu$ and the shear modulus $\mu$, which is a function of $E$ and $\nu$. The coefficient $\alpha \geq 0$ represents the mechanical coupling of the fluid pressure and the porous solid. The amount of fluid which can be forced into the medium by a pressure increment under conservation of the volume of the medium is expressed by $d_{0} \geq 0$. A measure of Darcy flow with respect to the pressure gradient is given by $k \geq 0$.

The presented system of equations is solved by using the finite element method based on the standard Galerkin discretization scheme. As a result, a displacement vector field and a vector of scalar pressure values are obtained, both defined in the nodes of the discretization domain (see Section 2.4). 


\subsection{Volume Reconstruction}

The optimization criterion for the quality of the elasticity parameters is the normalized mutual information function between the image reconstructed from the simulation and the intraoperative dataset. For this purpose, intensities in the deformed volume have to be recalculated in each iteration of the optimization. The direct result of the numerical simulation is a vector field of displacements defined only in vertices of a tetrahedral grid in the computational domain. Therefore, all tetrahedra in the deformed grid are traversed and for each element its inner voxels are considered. The displacement of each voxel is calculated as a scalar product of its barycentric coordinates and of the displacements given in the four nodes of the tetrahedron the voxel belongs to. Finally, a new intensity value is computed by moving back the voxel after the deformation and by trilinear interpolation of the intensities in the preoperative MR volume. This back-projection technique prevents holes in the reconstructed dataset.

\subsection{Geometry Generation and Boundary Conditions}

Prior to any optimization iteration, the brain surface is extracted from the preoperative MR data. A tetrahedral grid bounded by this triangular surface is then generated. For the numerical stability of further computations, special algorithms are applied to ensure regularity of the grid (for details refer to [14]).

In order to reflect the intraoperative settings in the numerical model, the forces which affect the brain during surgery have to be defined. In [16, non-rigid registration of MR images acquired before and after compressing a volunteer's breast was utilized to derive image-based forces. In our model, the load vector is set according to the location of the skull opening. Since this information is included in the intraoperative dataset only, a rigid registration of the pre- and intraoperative MR scan is performed [2]. In a 3D editor supporting the fusion of the preoperative brain surface and the intraoperative MR data, the respective outer part of the generated volumetric grid is manually marked (see Figure 10. Subsequently, a separate boundary condition is set for the defined surface region.

After the boundary conditions have been set, initial guesses for the parameters $E$ and $\nu$ are made and the optimization is started. In each iteration only one parameter is changed and the new volume resulting from the simulation is computed. The process terminates when the similarity function $\mathbf{S}_{\text {sim,intra }}$ reaches its maximum within a given precision. For the optimization Powell's direction search method was applied [11.

\section{$3 \quad$ Results}

In order to assess the quality of the presented method and to determine elasticity parameters for brain tissue, computational experiments were conducted with the biomechanical model described in Section 2.2 and with pairs of T1-weighted pre- and intraoperative MR data. All scans obtained with a Siemens Magnetom 


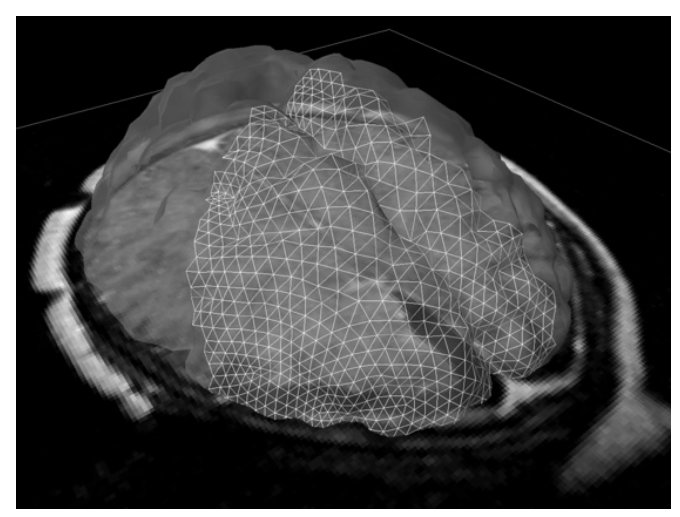

Fig. 1. The region for the boundary condition is manually marked in a 3D editor according to the location of the skull opening. The respective and defined part of the surface is visualized by displaying the underlying grid. The remaining brain surface is shown in a semitransparent representation

Sonata Maestro Class 1.5 Tesla scanner had a resolution of $512 \times 512 \times 160$ voxels and a voxel size of $0.49 \mathrm{~mm} \times 0.49 \mathrm{~mm} \times 1.0 \mathrm{~mm}$.

In the first experiment (Experiment 1), the numerical simulation was performed on a very coarse tetrahedral grid consisting of 9952 elements. The corresponding pre- and intraoperative datasets were rigidly registered and appropriate boundary conditions were set. $E$ and $\nu$ were initially set to $6500 \mathrm{~Pa}$ and 0.40 , respectively, while the parameter space was restricted (see Equation (2)) to the interval $\langle 1300,12000\rangle$ for $E$ and to $\langle 0.3,0.5\rangle$ for $\nu$. The corresponding penalty constant $c_{0}$ was set to -0.2 . The optimization terminated after 89 iterations (979 secs) and the maximal value of NMI was found at $\nu=0.452$ for the Poisson's ratio and $E=8863.01 \mathrm{~Pa}$ for the Young's modulus which corresponds to the value of $\mu=3052 \mathrm{~Pa}$ for the shear modulus (compare Equation (Ba)).

For the Experiment 2, a very fine grid was taken. The number of grid elements was 123496 . As in the case before, a rigid registration was performed to set proper boundary conditions. The initial values of all parameters were identical to those used in Experiment 1. The optimization returned the value of $\nu=0.461$ for the Poisson's ratio and $E=8196.21 \mathrm{~Pa}$ for the Young's modulus $(\mu=2805 \mathrm{~Pa})$ in 121 iterations, which took 9780 secs on a $2.66 \mathrm{GHz}$ PIV system. During both optimizations the precision (referring to the value of NMI) was set to $10^{-4}$.

Figure 2 shows the similarity function plotted retrospectively as a projection on one of the respective maximal parameters which allows visually assessing the result of both experiments.

\section{Discussion}

In the experiment performed on the coarse grid one global maximum was observed (see Figure $2 \mathrm{a}$ ). The resulting Poisson's ratio $\nu=0.452$ is very close to 


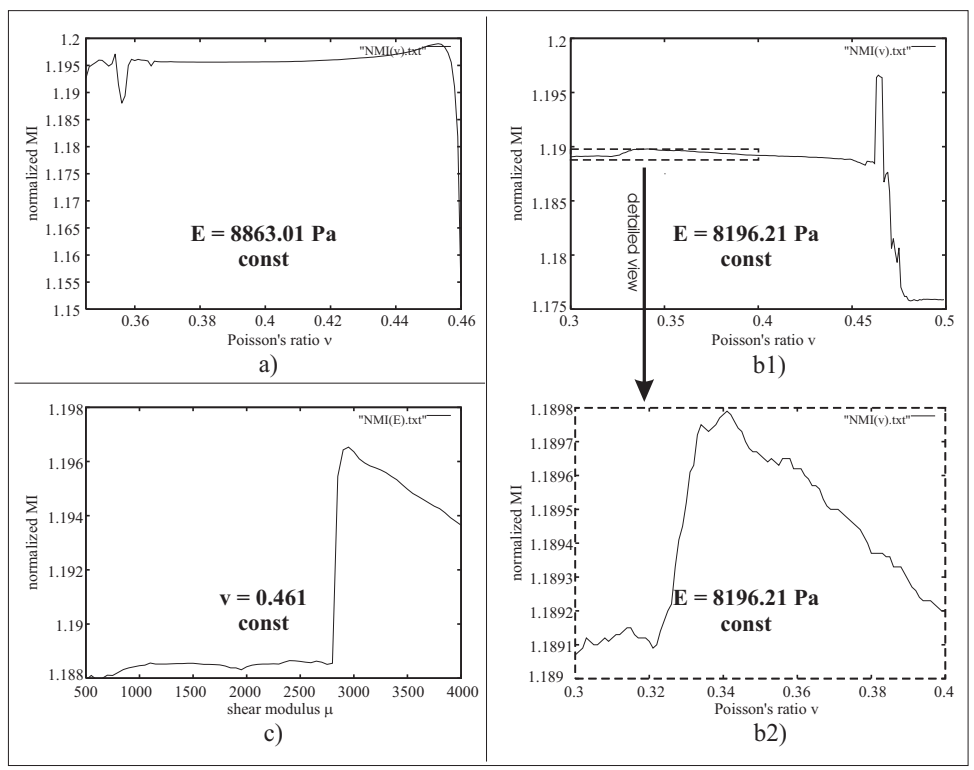

Fig. 2. Plot of the similarity function with one coordinate kept fixed: a) Experiment 1 (coarse grid) for $E=8863.01 \mathrm{~Pa}$. b1) Experiment 2 (fine grid) for $E=8196.21 \mathrm{~Pa}$. b2) Experiment 2 showing a detailed view of a local maximum in b1) with a differently scaled y-axis. c) Experiment 2, $\nu=0.461$ (here NMI as a function of $\mu$ )

the value reported in [7] whereas the corresponding Young's modulus is about 4 times bigger. Despite of the coarse grid, the simulation showed good convergence properties, except for $\nu$ in the interval $<0.3,0.357>$ (independent of the value of $E$ ). Nevertheless, the grid was too coarse to produce reliable results.

In the case of the fine grid, the computations exhibited a numerically more stable behavior. In the vicinity of $\nu=0.5$ for the Poisson's ratio, however, some problems with the numerical convergence were observed for individual values of $\nu$, which led to local fluctuations in the plot of NMI, see the small peaks in this region in Figure 2b1. The global optimum of $E=8196.21 \mathrm{~Pa}$ (see Figure 2k) for the Young's modulus is close to the value of $E=7425 \mathrm{~Pa}$ reported in 8 for the swine brain in an in vivo experiment. The corresponding value of $\nu=0.461$ for the Poisson's ratio is close to the values found in the literature [7] and to the result of Experiment 1.

In the experiment with the fine grid there exists also a local maximum at $\nu=0.341$ and $E=1775.48 \mathrm{~Pa}$ (see Figure 2b1 and 2 $\mathrm{b} 2$ ). A similar local minimum also appeared as a small peak around the value of $\nu=0.349$ in the first experiment with the coarser grid (see Figure $2 \mathrm{a}$ ). Although there are other approaches where $\nu=0.35$ is used as the elasticity parameter for the brain tissue [3], a reliable confirmation of the existence of such a local maximum requires further analysis since a bad convergence behavior of the numerical computations with our model on the coarse grid could be stated for $\nu \leq 0.357$. 


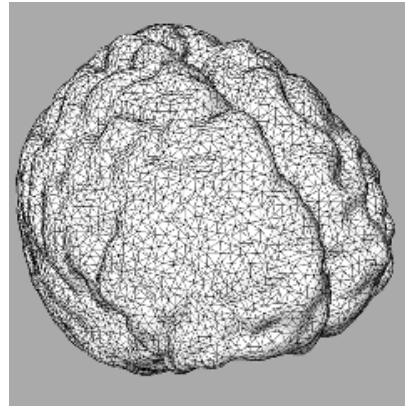

a)
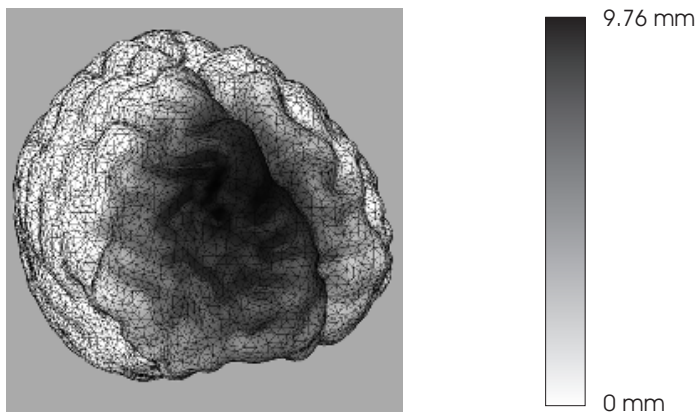

b)

Fig. 3. Surface visualization of the brain $a$ ) before and b) after volumetric deformation. Cortical displacements are coded with grey values. White means no displacement, black corresponds to the maximal displacement, in this case $9.76 \mathrm{~mm}$

In addition to the numerical analysis, a simulation with the use of the calculated optimal values $\nu=0.461$ and $\mu=2805 \mathrm{~Pa}$ was performed and the visual results of the deformation are presented in Figure 3

The mechanical properties of brain tissue obtained with the presented method contribute to a better physically-based simulation by improving the correspondence between the model and the real situation. The results obtained in the different experiments correspond very well to the values given in the literature [3,7]. This demonstrates the strength of the proposed strategy and shows that it is a promising alternative to other approaches where the mechanical properties of tissue are directly measured. Moreover, this technique has the advantage that the validation of the results is inherent.

\section{Conclusion}

A novel approach is presented that uses techniques of numerical simulation and registration for the determination of the mechanical properties of brain tissue. The conducted experiments proved the value of the method and the results correspond very well to the physical parameters in the literature. Thereby, our strategy contributes to solve the difficult problem of estimating the elasticity properties of living tissue. In the future, a statistical analysis will be conducted in order to analyze the obtained parameters.

Acknowledgments. This work was funded by Deutsche Forschungsgemeinschaft in the context of the projects Gr 796/2-1 - 796/2-3. We gratefully acknowledge Stefan Zachow (Zuse Institut Berlin) for his cooperation in the area of grid generation. 


\section{References}

1. A. Hagemann, K. Rohr, H.S. Stiehl, U. Spetzger, and J.M. Gilsbach. Biomechanical Modeling of the Human Head for Physically Based, Non-Rigid Image Registration. IEEE Trans Med Imaging, 18(10):875-884, 1999.

2. P. Hastreiter and T. Ertl. Integrated Registration and Visualization of Medical Image Data. In Proc. CGI, pages 78-85, Hannover, Germany, 1998.

3. M. Kaczmarek, R.P. Subramanian, and S. R. Neff. The Hydromechanics of Hydrocephalus: Steady-State Solutions for Cylindrical Geometry. Bull Math Biol, 59:295-323, 1997.

4. T.A. Krouskop, T.M.Wheeler, F. Kallel, B. Garra, and T. Hall. The Elastic Moduli of Breast and Prostate Tissues Under Compression. Ultrason Imaging, 20:151-159, 1998.

5. A. Manduca, D. S. Lake, S. A. Kruse, and R. L. Ehman. Spatio-temporal Directional Filtering for Improved Inversion of MR Elastography Images. Med Image Anal, 7(4):465-473, 2003.

6. M. Miga. A New Approach to Elastography Using Mutual Information and Finite Elements. Phys Med Biol, 48:467-480, 2003.

7. M. Miga, K. Paulsen, P. Hoopes, F. Kennedy, and A. Hartov. In Vivo Modeling of Intersitial Pressure in the Brain under Surgical Load Using Finite Elements. J. Biomech. Eng., 122:354-363, 2000.

8. K. Miller and K. Chinzei. Simple Validation of Biomechanical Models of Brain Tissue. In Proc. European Society of Biomechanics Conference, volume 31, page 104. Elsevier Science, 1998.

9. K. Miller, K. Chinzei, G. Orssengo, and P. Bednarz. Mechanical Properties of Brain Tissue In-vivo: Experiment and Computer Simulation. J. Biomechanics, 33(11):1369-1376, 2000.

10. J. Ophir, F. Kallel, T. Varghese, E. Konofagou, S. K. Alam, T. Krouskop, B. garra, and R. Righetti. Elastography. Comptes Rendus de l'Acadmie des Sciences - Series IV - Physics, 2(8):1193-1212, 2002.

11. W. H. Press, S. A. Teukolsky, W. T. Vetterling, and B. P. Flannery. Numerical Recipes in $\mathrm{C}++$. Cambridge University, New York, 2002.

12. L. Sandrin, M. Tanter, S. Catheline, and M. Fink. Shear Modulus Imaging with 2-D Transient Elastography. IEEE Trans Ultrason Ferroelectr Freq Control, 49(4):426435, 2002.

13. R. E. Showalter. Diffusion in Poro-Elastic Media. J Math Anal Appl, 251:310-340, 2000.

14. G. Soza, R. Grosso, P. Hastreiter, U. Labsik, Ch. Nimsky, R. Fahlbusch, and G. Greiner. Fast and Adaptive Finite Element Approach for Modeling Brain Shift. In Proc CURAC (Dt. Gesell. Computer and Robotorassistierte Chirurgie), 2003.

15. C. Studholme, D. L. G. Hill, and D. J. Hawkes. An Overlap Invariant Entropy Measure of 3D Medical Image Alignment. Pattern Recogn, 32(1):71-86, 1999.

16. C. Tanner, A. Degenhard, J. A. Schnabel, C. Hayes, L. I. Sonoda, M. O. Leach, D. R. Hose, D. L. G. Hill, and D. J. Hawkes. A Comparison of Biomechanical Breast Models: a Case Study. In Proc. SPIE Medical Imaging 2002, volume 4683, pages 1807-1818, 2002. 